ISSN: 0514-7336

DOI: http://dx.doi.org/10.14201/zephyrus201984161182

\title{
MORFOMETRÍA DE CERÁMICAS GRISES CATALANAS: ALGUNAS CONSIDERACIONES SOBRE LA DEFINICIÓN DE TIPOS EN CERÁMICA COMÚN DE ÉPOCA MEDIEVAL Y POSTMEDIEVAL
}

\section{Morphometric analyses of Catalonian common greywares: some thoughts on typological classification of medieval and post-medieval pottery}

\author{
Esther Travé Allepuz*, Guillem Domingo Ribas*, Joan Vicens Tarré** y Alfred Mauri Martí*** \\ * Dpto. de Historia y Arqueología. C/ Montalegre, 6. Facultad de Geografía e Historia. Univ. de Barcelona. 08001 \\ Barcelona. Correo-e: esther.trave@ub.edu; gdominri7@alumnes.ub.edu. ID ORCID: https://orcid.org/0000-0002- \\ 6769-4487; https://orcid.org/0000-0002-7848-1321 \\ ** Museu de la Terrissa de Quart. Plaça de la Vila, 2. 17242 Quart (Girona). Correo-e: jvicens@quart.cat. ID \\ ORCID: https://orcid.org/0000-0003-1080-1875 \\ *** Centre d'Estudis Martorellencs. Plaça de la Vila, 41.08760 Martorell (Barcelona). Correo-e: alfred.mauri@ \\ uab.cat. ID ORCID: https://orcid.org/0000-0002-9168-4385
}

Recepción: 21/02/2019; Revisión: 11/07/2019; Aceptación: 2/10/2019

\begin{abstract}
ReSUMEN: La cerámica común de cocción reductora y uso culinario constituye uno de los materiales arqueológicos más frecuentes en época medieval. A las dificultades de estudio inherentes a la gran homogeneidad de estas cerámicas, de fábricas bastas, irregulares, preferentemente urdidas y acabadas mediante un torneado lento, se les añade su elevada fragmentación y el volumen ingente de materiales recuperados en muchos contextos. En este trabajo presentamos los resultados obtenidos del análisis morfométrico de productos de alfarería tradicional de obra negra procedentes de Quart (Girona) y de cerámicas grises medievales del yacimiento de Santa Margarida (Martorell, Barcelona). A tenor de los resultados obtenidos demostramos la validez metodológica de una propuesta de clasificación que contempla la explotación estadística multivariante de datos morfométricos, incluyendo la variable del perfil de los vasos. Dicha explotación permite obtener clasificaciones tipológicas precisas que tienen en cuenta el grado de fragmentación y permiten definir tipos comparables.

Palabras clave: tipología; metodología; estadística; análisis vectorial; arqueometría; Quart; Santa Margarida.

AвsтRACT: Cooking wares fired under reducing atmosphere are one of the most common archaeological findings in medieval contexts. These are coarse, irregular and mostly coil-built vessels finished on the wheel. The great homogeneity of these products, together with the high level of breakage and the massive amount of sherds found out in many contexts are the main difficulties to deal with in order to study them. In this paper results obtained from the morphometric analysis of traditional craft greyware pottery from Quart (Girona) and medieval greywares from the site of Santa Margarida (Martorell, Barcelona) are introduced. According to the obtained results in both cases, we demonstrate the methodological validity of including vessel's profile measurement within a multivariate statistic exploitation of morphometric data. This exploitation allowed us to obtain precise classifications that take into account the degree of breakage and to establish comparable types.

Key words: typology; methodology; statistics; vectorial analysis; archaeometry; Quart; Santa Margarida.
\end{abstract}




\section{Introducción ${ }^{1}$}

La cerámica común, de carácter utilitario y cocción oxidante o preferentemente reductora sin decoración o con motivos incisos muy exiguos, es un elemento habitual en los contextos arqueológicos de la Cataluña medieval. Tras el declive de las grandes producciones estandarizadas de época romana y la desaparición de los productos tardíos o de imitación que perduran entre los ss. V y VII, se documenta un panorama cerámico fuertemente regionalizado, marcado por la presencia casi exclusiva de producciones de cocina, con pastas de factura basta, acabados sencillos y un repertorio formal muy limitado que se mantiene hasta los ss. XI y XII, en contextos cristianos. Cuando, con la eclosión del fenómeno urbano y el nacimiento de nuevas clases sociales, se generalicen la producción y el uso de nuevas vajillas vidriadas de mesa con motivos decorativos variables y una variedad formal y estilística cada vez más compleja, la producción común de cocina experimentará cambios trascendentales, como la aplicación sistemática de un vidriado funcional, sin fines decorativos. La cerámica de cocción reductora pervivirá, sin embargo, vinculada a nuevos usos de carácter doméstico multifuncional o en relación con las necesidades agrícolas y ganaderas, ampliando notablemente el espectro tipológico en todos los casos (Travé y Vicens, 2018). En los contextos arqueológicos, la homogeneidad de este tipo de cerámica dificulta enormemente los procesos de clasificación y seriación de unas producciones cuya distribución territorial y rasgos tecnológicos conocemos cada vez mejor a tenor de los estudios recientes

1 Este artículo se inscribe en la línea de investigación de Estudio de la Cerámica medieval y postmedieval y de los procesos productivos del Grupo de Investigación en Arqueología Medieval y Postmedieval de la Univ. de Barcelona (GRAMP.uB), consolidado por la Generalitat de Catalunya (2017sGR-833-GRC), del que son miembros todos los firmantes. El trabajo forma parte también del proyecto de investigación competitivo en materia de arqueología y paleontología titulado Projecte de recerca arqueològica integral al Priorat de Rocafort: jaciments de Sant Genis i Santa Margarida (Martorell), 2018-2021 (2018/035) financiado por la Generalitat de Catalunya y el Ayto. de Martorell.
(Travé, 2018). El estudio de este tipo de cerámicas requiere de una aproximación metodológica en la que podamos integrar con cierta precisión el componente morfológico o tipológico de los individuos a estudiar en un ejercicio de cuantificación como los que conocemos para los contextos cerámicos más antiguos (Adroher et al., 2016; Poblome y Bes, 2018) o para cronologías medievales y modernas (Escribano, 2017; Busto-Zapico, 2015).

Estas propuestas de estudio pasan por poner en relación las fábricas determinadas a partir de un análisis macro- o microscópico de pastas, con los acabados de superficie o las formas cerámicas con sus variantes más o menos numerosas, entendiendo que el resultado de esta integración de múltiples variables es el que define producciones que, en su sentido más amplio, reflejan las capacidades técnicas, elecciones de carácter sociocultural, preferencias o gustos de las comunidades del pasado (Poblome y Bes, 2018: 732). En cualquier caso, la noción de serie o tipo, entendido como un conjunto de valores morfológicos que tradicionalmente han sido utilizados para distinguir piezas diferentes, constituye una variable más de la cuantificación, partiendo de la base de que los tipos vienen predeterminados en cada clase cerámica por los estudios que se hayan realizado en torno a ella (Adroher et al., 2016: 104). No se aborda en los protocolos más recientes cómo o por qué razones un tipo determinado incluye unos fragmentos o formas y no otros, cuestiones que son objeto de un intenso debate (Contreras, 1984: 335336; Hamilakis y Jones, 2017: 79; Nail, 2017).

Aceptando los distintos planteamientos cuantitativos vigentes, en contextos medievales y especialmente en el ámbito de las producciones comunes de carácter utilitario, la definición de tipos $-y$ por ende su cuantificación- es especialmente compleja. Lo es, en primer lugar, porque contamos con elementos de referencia escasos y, en segundo lugar, porque los estudios tipológicos realizados para la cerámica reductora de época medieval en Cataluña en contextos rurales entre los ss. VIII y XII tienden a definir a grandes rasgos y casi en exclusiva una morfología que diferencia entre formas abiertas y cerradas, con una predominancia de ollas y algunos 


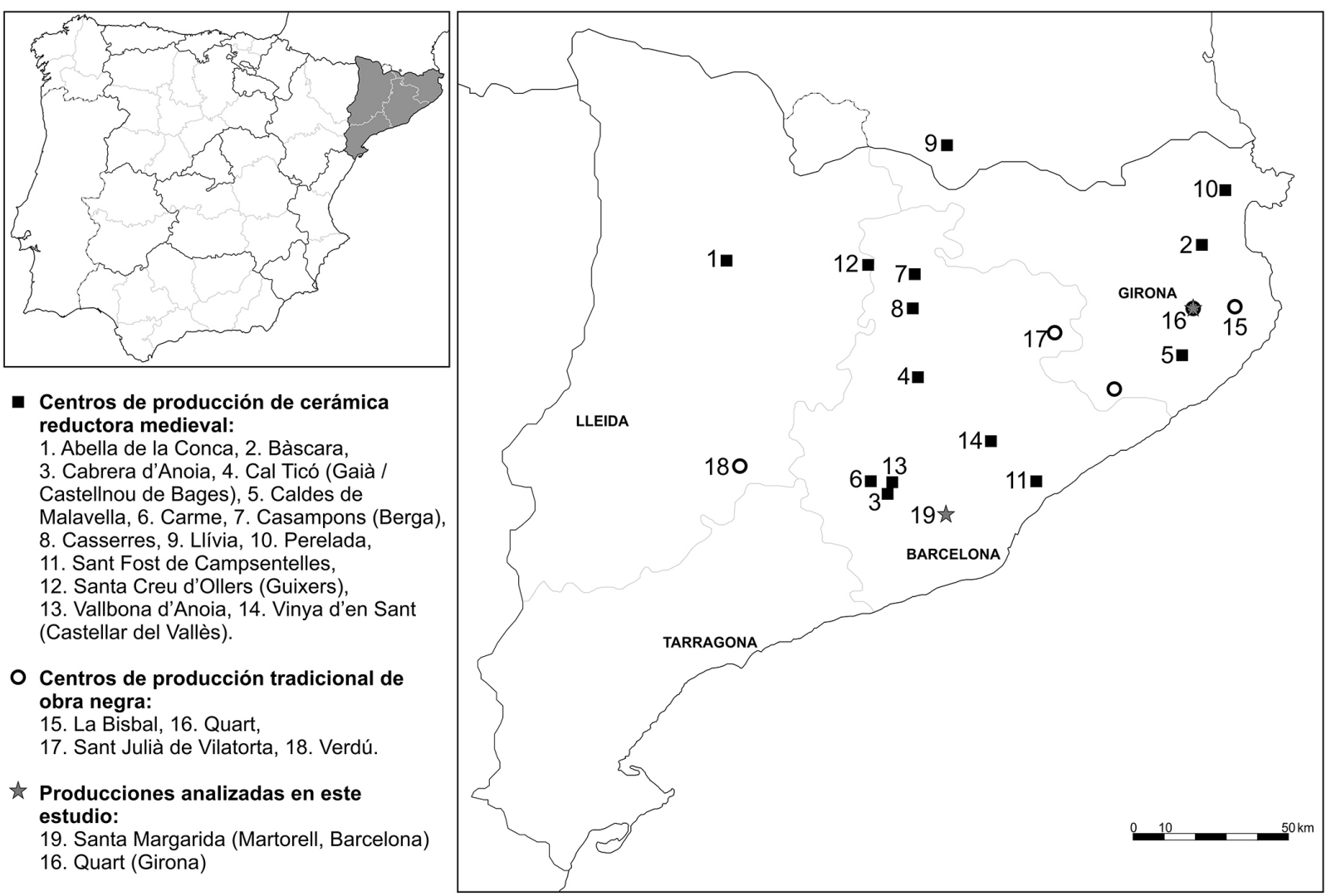

FIG. 1. Mapa de localización de los conjuntos cerámicos que son objeto de este estudio en relación con el panorama conocido de la producción de cerámicas grises catalanas en época medieval y postmedieval.

otros enseres de cocina, que con carácter secundario completan el repertorio (Riu, 1984; González, 1997), siendo muy escasos los estudios que intentan profundizar algo más en dicha simplicidad ${ }^{2}$. A ello se le añade, además, la regionalización de los circuitos de distribución, que se mantiene también en los siglos posteriores y que también constituye una dificultad añadida al estudio.

En este trabajo queremos presentar una propuesta de análisis morfológico consistente en definir la tipología de un conjunto cerámico determinado a partir de una explotación estadística de datos morfométricos considerando un parámetro singular que hemos denominado "vectorización de líneas curvas" ${ }^{3}$. Esta técnica pretende incorporar en el análisis

2 Travé, E. (2009): Producció i distribució d'una terrisseria medieval: Cabrera d'Anoia. Tesis doctoral presentada en 2019 en la Univ. de Barcelona.

3 Travé, op. cit. n. 2, p. 217. morfométrico de manera simultánea las variables relativas al grado de fragmentación de las piezas y la forma de los perfiles. Fue inicialmente desarrollada a partir de una propuesta anterior (Llanos y Vegas, 1974), reformulada a partir de otras aproximaciones (Orton, 1988: 46) y en los últimos años ha sido aplicada a distintos conjuntos cerámicos de época medieval facilitando la definición tipológica de los

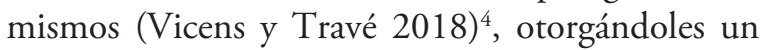

4 También sobre ello Travé, E.; Navarro, R. y Socorregut, J.: "Pottery Production and Importation at the Medieval Town of Martorell (Barcelona, Spain): New data and methodological perspectives for a chronological review through the petrographic and morphometric analysis. Poster". Presentado en Ceramic Petrology Group Annual Meeting (Tübingen 2018); Ollich, I.; Travé, E.; Ocaña, M.; Pratdesaba, A. y Rocafiguera, M. (2019): "La ceràmica medieval de l'Esquerda. Aproximació tipològica i arqueomètrica”. En Homenatge al Dr. Alberto López Mullor. Estudis sobre ceràmica i arqueologia de l'arquitectura. Barcelona, en prensa. 
componente ańadido de objetivación sobre la mera identificación visual de tipos y refinando los procesos de muestreo con finalidades analíticas (Mauri et al., 2012) .

A fin de demostrar su eficacia y validez, discutimos los resultados obtenidos en el estudio de dos conjuntos cerámicos con rasgos complementarios: por un lado, una colección de 65 piezas enteras de cerámica tradicional procedente del municipio de Quart, Girona (Vicens y Travé, 2018); y, por otro, 120 fragmentos con forma procedentes de cuatro silos medievales, pero no coetáneos, excavados en el yacimiento de Santa Margarida en Martorell, Barcelona (Travé et al., 2018) ${ }^{6}$ (Fig. 1). El objetivo principal de nuestro trabajo es testar cuáles son los criterios predominantes en la definición de grupos a partir de una explotación estadística multivariante de los datos y qué aplicaciones o resultados puede tener en la definición tipológica en conjuntos muy homogéneos con distintos grados de fragmentación. Sin obviar el carácter subjetivo de los muestreos y la disparidad de aproximaciones en las distintas prácticas cuantitativas habituales (Escribano, 2017: 292), buscamos un equilibrio entre lo ideal y lo posible, incorporando un criterio de objetivación en la determinación tipológica de las producciones de cerámica gris medieval y postmedieval que puede ser de utilidad en otros conjuntos con problemas de base similares, especialmente cuando nos hallamos ante centenares de individuos fragmentados muy parecidos entre ellos.

5 Además, Travé, E.; Mauri, A.; Navarro, R. y Del Fresno, P.: "Sampling strategies via statistical exploitation of morphometric data: Maximizing the assets of petrographic examination for a chronological review at the medieval town of Martorell (Barcelona, Spain)". Póster presentado en EMAC-2019. European Meeting on Ancient Ceramics. Barcelona.

6 Travé, E.; Navarro, R.; Mauri, A.; Farreny, A.; Del Fresno, P. y Socorregut, J.: “De l'església paleocristiana a la sagrera medieval: Transformacions estructurals i ordenament intern del Jaciment de Santa Margarida (Martorell, Barcelona)". En El cristianisme en l'antiguitat tardana. Noves perspectives. Actes del $4 t$ Congrés Internacional d'Arqueologia del Món Antic Tarraco Biennal. VII Reunió d'Arqueologia Cristiana Hispànica. Tarragona: Univ. Rovira i Virgili, en prensa.

(C) Universidad de Salamanca

\section{Materiales y método de estudio}

Ciertamente, el grado de fragmentación de los materiales condiciona notablemente las posibilidades y la resolución de los estudios al respecto en contextos arqueológicos. Con independencia de las estrategias cuantitativas que se lleven a cabo para minimizar su impacto (Mateo y Molina, 2016; Busto-Zapico, 2015; Escribano, 2017: 293; Orton et al., 1993), el resultado final del muestreo arqueológico que nos lleva a definir una tipología concreta es el de un conjunto fragmentado y no podemos obviar que esa realidad es un condicionante de la determinación tipológica. Nuestra propuesta parte de una definición de grupos según la explotación estadística de un número determinado de variables especialmente significativas y que considere también el grado de fragmentación de los materiales en su estudio formal y en ello juega un papel especial el perfil del fragmento conservado.

Las propuestas de automatización más recientes que incorporan el perfil de los vasos (Gilboa et al., 2004; Karasik y Smilansky, 2011) suelen focalizar la atención precisamente en ese perfil de las piezas a veces en exclusiva y no en el conjunto de variables o atributos - por ejemplo, los diámetros, altura o grosor de las paredes- que pueden determinar una forma concreta (Contreras, 1984: 328), por lo que planteamos nuestra propuesta como una herramienta útil para la clasificación formal de materiales cerámicos. Los resultados obtenidos hasta el momento en ensayos previos (Vicens y Travé, 2018, Travé et al., 2018) han sido satisfactorios, pero se hace necesario comprobar que, efectivamente, integrar la variable derivada de la vectorización del perfil influye siempre de la misma manera o de maneras comparables en un análisis multivariante. De ahí la selección de los dos estudios de caso que presentamos.

Las 65 piezas de Quart son representativas de una tipología bien conocida en los contextos tradicionales, en donde se ha mantenido una producción de cerámica con unas formas que han experimentado muy escasas variaciones desde el s. Xvi (Soler, 1985 y 2009). Estas han sido deliberadamente 

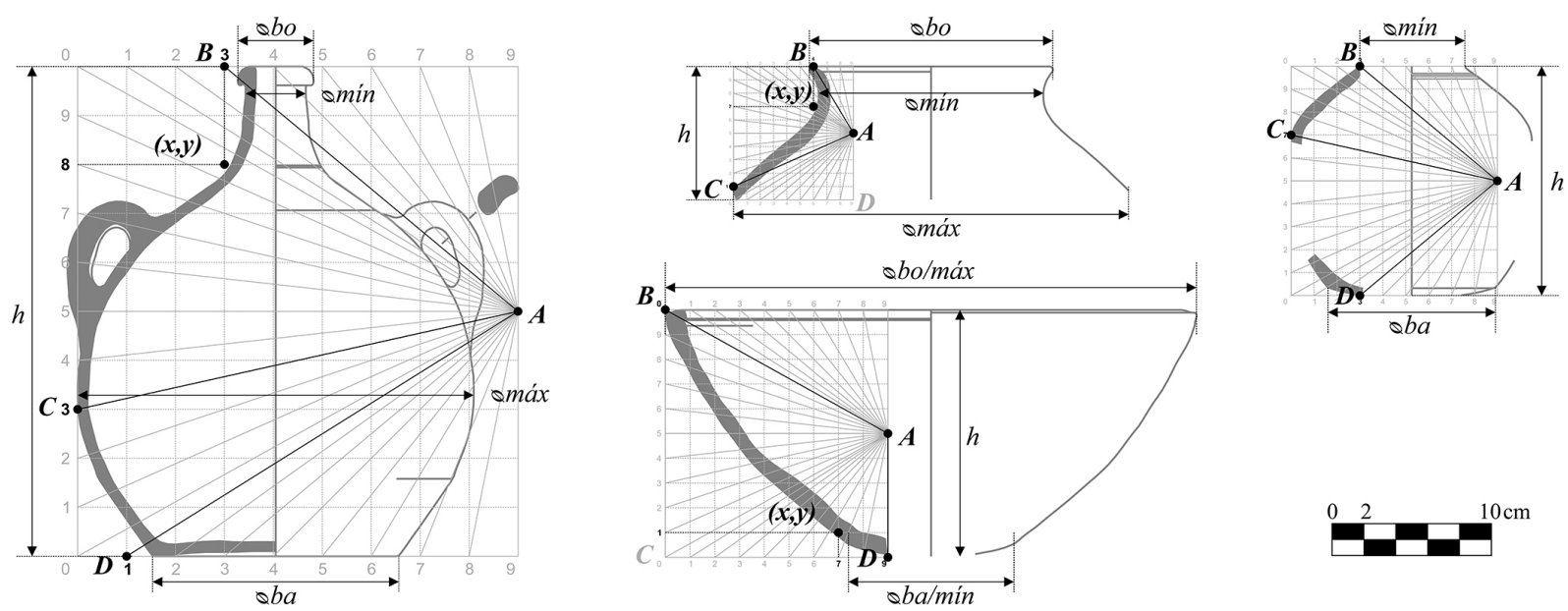

FIG. 2. Distintos ejemplos de mediciones del perfil de vasos cerámicos a partir del método de vectorización de líneas curvas.

seleccionadas, entendiendo el tipo de la manera más restrictiva posible, a fin de testar el potencial de la explotación estadística de datos morfométricos incluyendo la vectorización de perfiles al margen de la fragmentación, es decir, en material entero, con el objetivo de observar las mediciones de una serie de parámetros característicos de cada tipo y definir con precisión las variaciones de cada uno (Baxter, 2006). Esta manera de proceder no es nueva y en los estudios en que se ha puesto en práctica (Arcelin-Pradelle y Laubenheimer, 1985; Opgenhaffen et al., 2018) ha resultado muy reveladora. Debe entenderse que una muestra representativa de material entero con este fin necesariamente debe ser experimental o subactual -como es nuestro casodada la imposibilidad de conseguirla en contextos arqueológicos.

La selección de los materiales procedentes de Santa Margarida, por el contrario, parte de una realidad distinta: el conjunto incluye la totalidad de fragmentos con forma -120- de individuos diferenciados entre un conjunto de 955 fragmentos que ha sido objeto de un estudio macroscópico y posterior caracterización petrográfica. En este caso, trabajamos con material arqueológico obtenido de contextos excavados, altamente fragmentado, analizado sin selección previa en el seno de cada contexto, es decir, realizamos el ejercicio de clasificación partiendo desde cero tal como es habitual en la práctica arqueológica, considerando en nuestro caso el número total de restos $-\mathrm{NR}-\mathrm{y}$ calculando el número mínimo de individuos -NMI- como principal estrategia cuantitativa (Solaun, 2005: 36-37). En función de la clasificación formal obtenida y del análisis macroscópico de pastas, la caracterización petrográfica de la cerámica de estos silos se ha llevado a cabo a partir de un muestreo mucho más refinado que ha permitido maximizar la interpretación de los resultados. Dicho estudio de caso nos permite ilustrar la aplicación de la técnica en contextos arqueológicos habituales y discutir el potencial de la misma.

En ambas colecciones se han documentado gráficamente los individuos a partir de un dibujo en alzado y sección a escala real. Sobre dicho dibujo se han realizado las mediciones de las variables siguientes: diámetro de boca y/o de base, diámetro mínimo y máximo del cuerpo, grosor mínimo, medio y máximo de las paredes, altura conservada -real, absoluta o restituida- y el perfil de la generatriz del vaso considerado a partir de su análisis vectorial (Mauri et al., 2012: 50-52). Este último parámetro pone en relación los grados de apertura y cierre de la boca de cada individuo en relación con la base o carena, el grado de esfericidad de la panza o el carácter más o menos exvasado de los bordes (Fig. 2) a partir de un cálculo sencillo sobre una plantilla predeterminada (Llanos y Vegas, 1974: 265-282). 
En el análisis de material no fragmentado, el cálculo establece la relación vectorial entre los cuatro diámetros determinados como variables -boca, base, mínimo y máximo- respecto a un punto fijo -A- situado en el punto medio de la altura $-\mathrm{h}-\mathrm{y}$ a una distancia de $0,9 \mathrm{~h}$ respecto de un punto cualquiera de la circunferencia que corresponde al diámetro máximo. La medición es sencilla puesto que se obtiene a partir de la superposición sobre el dibujo de una plantilla a partir de la cual se identifican tres puntos $-\mathrm{B}$, C y D- que corresponden respectivamente al extremo de tres vectores determinados por la relación entre los puntos de inflexión del perfil del vaso y el punto A, y una coordenada cartesiana $-\mathrm{x}, \mathrm{y}-$ que corresponde a un cuarto punto de inflexión. De este modo se obtiene una expresión del tipo BCYXD ${ }^{7}$.

En los conjuntos de material fragmentado la explotación de dicho cálculo permite, como tendremos ocasión de mostrar, agrupar los materiales en función del grado de fragmentación, por lo que facilita la ordenación y el estudio del conjunto. Es decir, se ha determinado cada tipo a partir de fragmentos comparables, representativos de una misma parte proporcional de la forma completa y no al revés. Los resultados obtenidos en ambos casos, estructurados en forma de tabla ordenada, con las variables almacenadas en columnas y las observaciones en filas (Wickham, 2014), han sido explotados estadísticamente, mediante múltiples herramientas de uso habitual ampliamente consolidadas en arqueología (Contreras 1984: 353-370; Baxter, 1995; Aitchinson, 1986), analizando la estructura interna de los datos y definiendo los grupos o tipos, en base al mayor número de variables y representatividad posibles.

Tras obtener las tablas de datos morfométricos anteriormente descritas, se ha realizado un análisis exploratorio de los datos -EDA- para todas las variables en ambos conjuntos. Este paso inicial es necesario para la identificación de posibles relaciones entre las variables estudiadas. Considerando la complejidad de trabajar con dos conjuntos de datos distintos, el análisis exploratorio nos ha permitido

7 Travé, op. cit. n. 2, pp. 217-231. identificar relaciones y patrones significativos, así como proponer nuevas hipótesis de trabajo a partir del reconocimiento de relaciones previamente desconocidas.

Para una mejor representación e interpretación de los resultados, hemos optado en primer término por el análisis de componentes principales -ACP-. Este análisis implica la transformación lineal de variables correlacionadas a variables no correlacionadas por pares que maximizan la variabilidad de un número pequeño de componentes. Así se consigue pasar de un número elevado de dimensiones a un pequeño número de valores calculados como combinaciones de las variables iniciales. De este modo, a partir de los primeros tres o cuatro componentes extraídos, es posible realizar gráficos de dispersión de doble o triple entrada para explorar la estructura de los datos (García-Jiménez et al., 2000; Dillon y Goldstein, 1984). Este tipo de gráficos siempre explica una varianza mayor que cualquier otro realizado sobre los valores originales.

Los ACP se han practicado de manera doble para cada conjunto de datos: sobre las mediciones originales calculadas en milímetros, así como la combinatoria de cinco cifras que define la vectorización del perfil, por un lado; y también sobre una transformación logarítmica de base 10, que equipara en cierta medida los valores, reduce la variabilidad del conjunto y permite definir la estructura de los datos con mayor precisión. La comparación de ambos resultados es la que nos ha permitido definir grupos morfológicos en todos los casos. Hemos observado que trabajar con datos crudos es especialmente útil en los ACP realizados tras la extracción de muestras aisladas o de grupos bien definidos, en donde la variabilidad entre las muestras restantes ya es muy baja en origen, mientras que los datos logarítmicos funcionan bien al principio, cuando el conjunto incluye muchas variantes tipológicas.

Sobre la necesidad o no de convertir o estandarizar los datos existe un cierto debate en el campo del análisis de datos composicionales y la literatura al respecto es muy abundante (Aitchinson, 1986; Baxter, 1995; Aitchinson y Greenacre, 2002; Baxter y Freestone, 2006; Buxeda, 2008). En el caso de 



FIG. 3. Tipología de la alfarería tradicional de Quart. Enseres de cocina: ollas altas (oa); baja (ob) y castañera (oc) todas ellas de obra roja; olla de cuerpo esférico (oe) de obra negra; pucheros (ansats) de obra roja (A1) y negra (A2). Recipientes para la contención de líquidos: alcuza (càntir d'oli) (CO); barrales (B1 y B2) para agua o vino; cántaros (C) y cántaro de pescador (CP); cántaro de pozo, con boca y asa superiores (poal) (P); tinaja para líquidos (doll) (D) y medidas de vino y/o aceite (mallal) (Ma) y tramostera (Tr). Vasijas de almacenaje y usos domésticos: jarras (G), brasero (maridet) (Br) y barreño de grandes dimensiones (cossi) típico de Quart (Co). 
datos morfométricos, no se han llevado a cabo estudios similares hasta el momento, por lo que sugerimos aplicar unos parámetros de cálculo amplios, mediante múltiples análisis descartando o añadiendo variables y analizando a fondo la estructura interna de los datos antes de tomar partido por una rutina concreta.

De hecho, la explotación estadística no es sustitutoria de la definición de hipótesis de trabajo previas construidas a partir de la determinación de grupos macroscópicos que deben validarse; ni tampoco debe utilizarse para validar hipótesis que la misma explotación ha contribuido a generar, a riesgo de entrar en una argumentación circular de carácter tautológico (VanPool y Leonard, 2011: 39). Explorar la estructura interna de los datos previo a la determinación de grupos/tipos es especialmente útil para refinar los resultados y testar su validez como elementos significantes en términos históricos o antropológicos que sean determinantes y explicativos de transformaciones ocurridas, por ejemplo, en las preferencias sociales de consumo o en las técnicas de producción.

Con el fin de comparar ambas colecciones se han realizado matrices de correlación, que son especialmente útiles en esta exploración de variables con independencia de los grupos generados, dado que dichas representaciones sintetizan las relaciones entre las variables empleadas en el estudio y facilitan su definición e influencia en una explotación multivariante de datos. En base al coeficiente de correlación, la matriz resultante también destaca las diferencias entre un conjunto de piezas enteras y fragmentadas y nos permite evaluar el impacto de la variable de vectorización del perfil en ambos casos. Para los análisis estadísticos y la creación de representaciones gráficas, hemos utilizado el software IBM SPSS en su versión 19.0 y también R 3.5.1 ( $\mathrm{R}$ Core Team, 2018). La creación de las matrices de correlación se ha llevado a cabo mediante el paquete corplot (Wei y Simko, 2017) ${ }^{8}$.

8 Para posibles réplicas de los análisis y gráficos propuestos, se ha publicado una copia del código bajo Licencia Pública General de GNU (https://github.com/gdomrib/ stats/tree/master/morfometria_sm_quart).

\section{Dos estudios de caso: Quart (Girona) y Santa Margarida (Martorell, Barcelona)}

\subsection{Resultados de la clasificación morfométrica sobre un muestreo representativo de la tipología de Quart}

Quart (Girona) es el único municipio catalán que mantiene en la actualidad la producción de alfarería tradicional de obra negra como elemento emblemático y genuino, junto con otras producciones más frecuentes de obra roja comunes a algunos otros centros de la zona. La colección estudiada procede de los fondos del Museu de la Terrissa de Quart e incluye los principales tipos documentados entre las producciones tradicionales que se elaboran en el municipio desde el s. Xvi. Entre ellos se cuentan grandes contenedores para el almacenaje, medida y trasiego de líquidos -agua, vino o aceite-, casi siempre de obra negra a excepción de algunos cántaros denominados de invierno (Sempere, 1985: 35 ), vidriados y de obra roja; y algunos enseres para el fuego, varios tipos de ollas y pucheros de tamaños variables, también de uso culinario, en este caso de obra roja. No hemos querido obviar una de las formas más características de Quart -los cossis- o barreños de muy grandes dimensiones (Fig. 3), a pesar de que su identificación tipológica es muy clara aun en contextos fragmentados. Los incluimos precisamente para comprobar como determinados tipos con unas diferencias mucho más marcadas respecto del conjunto pueden modificar o condicionar la clasificación (Hamilakis y Jones, 2017: 80-81).

Sobre esta muestra se ha realizado un primer análisis de componentes principales - $\mathrm{ACP}-$ sobre una transformación logarítmica de base 10 , incluyendo la totalidad de las muestras y considerando todas las variables, que representamos en dos gráficos de doble entrada en función de los componentes resultantes 1, 2 y 3, comparados dos a dos (Fig. 4a-b). Este análisis proporciona una primera ordenación de los datos a partir de la cual se pueden advertir algunos aspectos significativos respecto de su estructura. El primero de ellos es la buena correlación de los resultados con la ordenación tipológica conocida, aun cuando la presencia de formas

Zephyrus, LXXXIV, julio-diciembre 2019, 161-182 

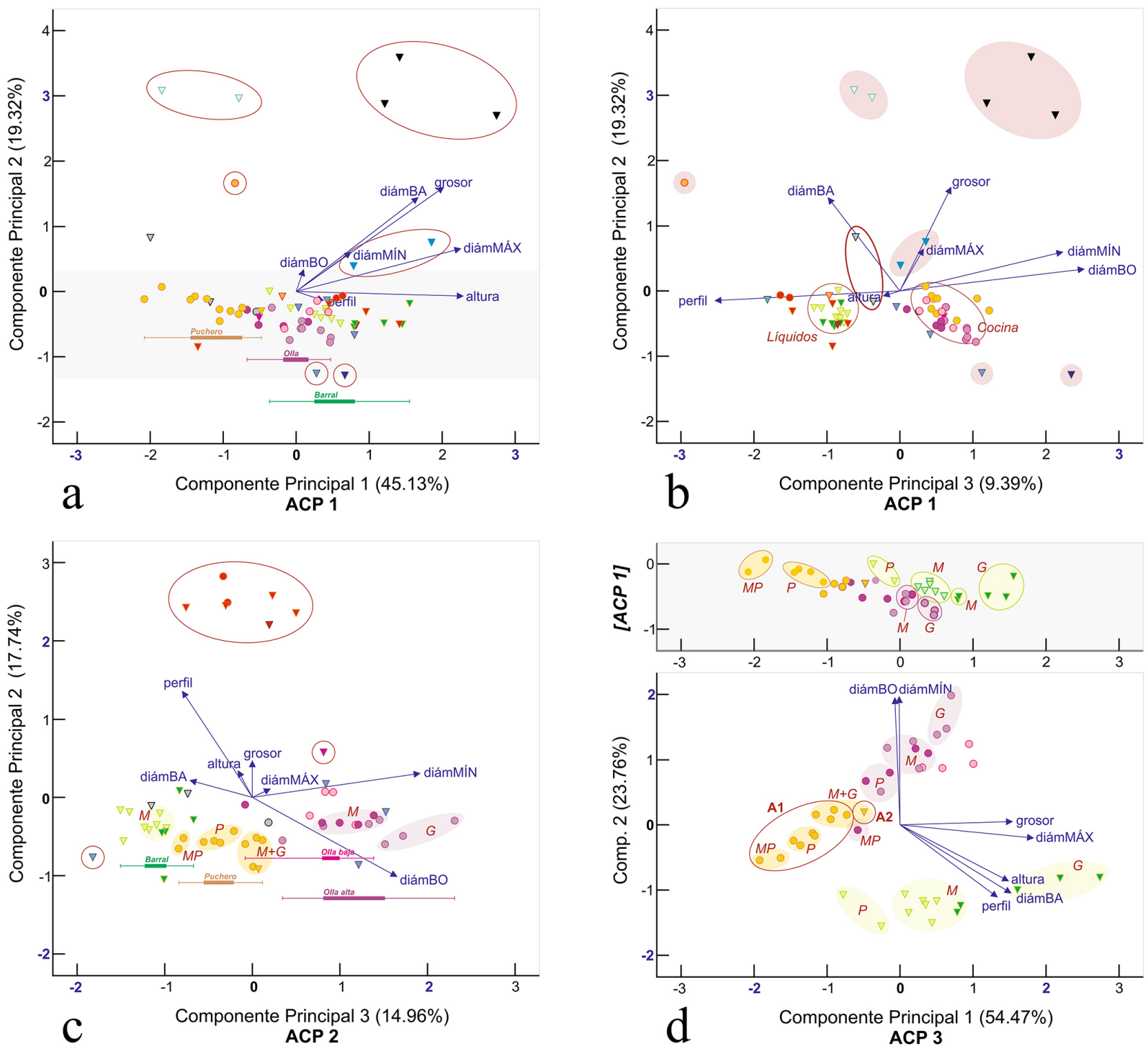

\begin{tabular}{|c|c|c|c|c|c|}
\hline $\begin{array}{l}\text { GRUPOS MORFON } \\
\text { Obra negra: } \\
\text { Alcuza (càntir d'oli) } \\
\text { Barral - B1 } \\
\text { Barral - B2 } \\
\text { Barreño (cossi) } \\
\text { Brasero (maridet) }\end{array}$ & $\begin{array}{l}\text { TRICOS } \\
\nabla \text { Cántaro } \\
\nabla \text { Cántaro de pescador } \\
\nabla \text { Cántaro de pozo (poal) } \\
\nabla \text { Jarra } \\
\nabla \text { Medida de aceite (tramos }\end{array}$ & $\begin{array}{l}\nabla \text { Medida de vino (mallal) } \\
\nabla \text { Olla esférica (bigatana) } \\
\nabla \text { Puchero (ansat) } \\
\nabla \text { Tinaja (doll) } \\
\text { tera) }\end{array}$ & $\begin{array}{l}\text { Obra roja: } \\
\text { Puchero (ansat) } \\
\text { - Cántaro de invierno } \\
\text { - Cántaro de pescador } \\
\text { - Castañera }\end{array}$ & $\begin{array}{l}\text { - Olla alta } \\
\text { Olla baja }\end{array}$ & $\begin{array}{l}\text { Abreviaturas tamaño: } \\
G=\text { Grande } \\
M=\text { Mediano } \\
P=\text { Pequeño } \\
M P=\text { Muy pequeño }\end{array}$ \\
\hline
\end{tabular}

FIG. 4. Gráficos de doble entrada que comparan por pares el valor de los distintos componentes resultantes de los ACP practicados sobre los datos morfométricos de la cerámica producida en Quart. Un primer ACP, con las muestras identificadas según la forma, permite aislar los tipos singulares en función de los dos primeros (a) o de los dos segundos componentes (b); segundo ACP calculado a partir de la omisión de los tipos singulares, con las muestras también identificadas según su forma (c); y tercer ACP calculado para las formas barral, puchero y olla (d), que permite la distinción de tamaños o subtipos.

especialmente características y morfológicamente muy distintivas como los barreños, cántaros de pozo o tinajas, representados además en un número reducido, contribuye a minimizar en gráficos de este tipo la variabilidad interna de otros grupos con mayores similitudes entre ellos. 
La comparación de valores obtenidos para los tres primeros componentes con las variables que mayor influencia ejercen en la determinación de los mismos también es significativa. En la determinación de los componentes 1 y 2 , que juntos explican un $64,45 \%$ de la varianza total del conjunto, podemos observar como las principales diferencias entre grupos vienen determinadas principalmente por su diámetro máximo y de base, el grosor de las paredes y la altura, variables todas ellas que acaban condicionando el tamaño de la pieza, mientras que la variable perfil determinada a partir de la vectorización no ejerce una influencia significativa en la determinación de tipos cuando las formas están enteras, al menos aparentemente, en función de los dos primeros componentes. Tampoco los diámetros mínimos o de borde parecen determinantes. Sin embargo, estas variables son especialmente significativas en la distinción funcional de los contenedores de líquidos y los enseres de cocina determinada a partir del tercer componente, a pesar del escaso porcentaje -9,39\%- de varianza que este explica (Fig. 4b). Del mismo modo, en la comparativa del primer y segundo componente, el tamaño de las piezas enteras actúa en detrimento de la forma en una clasificación morfométrica como la que nos ocupa en particular cuando la muestra incluye piezas de muy grandes dimensiones como los barreños o tinajas. El resultado es que los tipos con mayor representación -olla, puchero, barral y cántaro- quedan condicionados por su tamaño en la clasificación general determinada por el primer componente, con unos valores comprendidos entre - 1 y 0 para el segundo, junto con algunos tipos de dimensiones similares - medida de vino o jarra-, representados por un número escaso de muestras que no aparecen claramente aisladas en el gráfico general.

Por ello, se procede a realizar un segundo ACP a partir de los tipos más homogéneos, incluyendo todas las muestras con valores comprendidos entre -1 y 0 para el segundo componente. Dicha selección excluye automáticamente los tipos aislados por su singularidad o dimensiones. Los resultados del nuevo análisis (Fig. 4c) aíslan de manera evidente el grupo de los cántaros, especialmente por su perfil, y contribuyen a singularizar también los tipos menos representados. Al trabajar con una muestra más reducida

(C) Universidad de Salamanca y con una variabilidad interna menor, el porcentaje de varianza que explica cada componente presenta porcentajes muy similares entre los componentes segundo $-17,74 \%-$ y tercero $-14,96 \%-$, por lo que son especialmente útiles para singularizar tipos y, en cada uno de ellos, tamańos o tallas para las distintas muestras, máxime cuando ninguno de los dos está especialmente afectado por las variables altura y diámetro máximo, que son determinantes del tamaño.

Un último análisis practicado sobre datos crudos incluye únicamente las formas barral, puchero y olla, claramente distinguibles en función de todas sus variantes (Fig. 4d) a partir de los dos primeros componentes, que explican un $78,26 \%$ de la varianza. En este caso, puede observarse como las distintas variables ejercen una influencia parecida en la segregación del conjunto y cuáles son aquellas que contribuyen más a la determinación de un tipo u otro. Al comparar la distribución con la del primer análisis e identificar la posición de cada muestra en uno y otro gráfico, se observa cómo recipientes de capacidad similar -barrales pequeños y ollas medianas; o barrales medianos y ollas grandes- se agrupan de manera conjunta en el primer $\mathrm{ACP}$, pero se distinguen en el tercero.

Otro aspecto relativo a la clasificación morfométrica de estas producciones es determinar hasta qué punto es aceptable un cierto grado de variabilidad interna determinado por el tamaño en el seno de cada tipo y hasta qué punto se puede establecer una identificación o correlación entre posibles subtipos y la existencia de tallas predeterminadas. En este sentido, las fuentes documentales avalan para el caso de Quart una regulación al respecto a partir de 1703 que detalla específicamente las dimensiones de los distintos enseres fabricados en los talleres locales (Soler, 1985: 40-41). Una manera de determinar la regularidad y el carácter deliberado o no de las variaciones es comparar la correlación entre la adecuación de aquellas variables que determinen con mayor fiabilidad el tamaño con la distribución normal de las mismas (Arcelin-Pradelle y Laubenheimer, 1985: 134-135). De manera teórica podemos establecer que la distribución real de los valores en un mismo tipo debería ajustarse a la distribución normal (Fig. 5). 

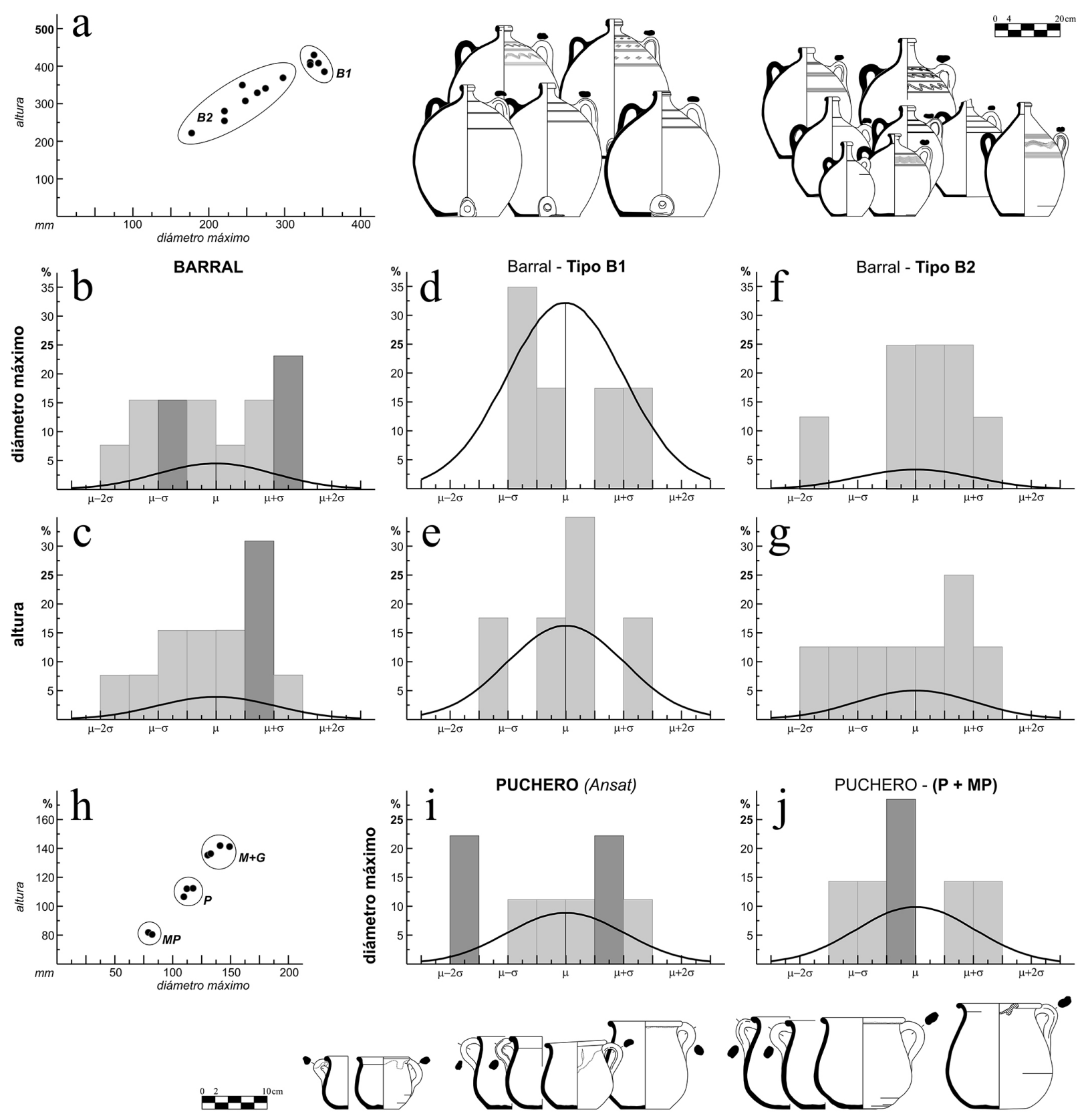

Fig. 5. Cuadro comparativo de las variables diámetro máximo y altura para las formas barral y puchero en base a su distribución normal e histogramas porcentuales absolutos. Gráfico bivariante de las variables diámetro máximo y altura del grupo de barrales (a) e histogramas comparados con la distribución normal para cada variable en función del grupo $(b, c)$ y de los dos tipos detectados $B 1(d, e)$ y $B 2(f, g) ; y$ mismo gráfico bivariante para la forma puchero $(b)$, con la comparación de histogramas y distribuciones para la variable diámetro máximo de todo el conjunto (i) y de los tamaños menores (j).

Tomando como ejemplo la forma barral, cuya distribución presenta una cierta variabilidad interna en los ACP, y analizando la distribución de dos variables fuertemente correlacionadas (Fig. 5a) e indicativas del tamaño en formas enteras como la altura y el diámetro máximo, puede observarse cómo 
para una curva de distribución normal muy poco pronunciada $-\mathrm{y}$, por tanto, con una gran variabilidad de los datos en ambas variables- la distribución real del muestreo presenta una distribución bimodal en la variable diámetro máximo, con un número de valores significativo por encima y por debajo de la media $-\mu$ - respectivamente (Fig. 5b). Asimismo, la variable altura presenta una distribución real con estructura unimodal, pero con una moda muy alejada de la media -casi una vez la desviación típica, $\sigma-$ (Fig. 5c). Dicha estructura de los datos es indicativa de la existencia como mínimo de dos tamańos diferenciados con entidad suficiente para considerarlos tipos per se.

Aislando los individuos de mayores dimensiones -Tipo в1-podemos ver que todos ellos cuentan además con una vertedera tubular en la base y un cuerpo ovalado característico. En este caso, la curva de distribución normal de las variables anteriores es mucho más pronunciada y la distribución real de los datos se ajusta a la probabilidad, a pesar de su distribución no continua, debida al número limitado de individuos (Fig. 5d-e). El Tipo в2, por el contrario, es algo más heterogéneo: las curvas de variación normal son poco pronunciadas y, aunque la distribución real no presenta desajustes significativos (Fig. 5f-g), es posible la existencia de algunas variantes relativas al tamaño que deberían validarse a partir de una muestra con mayor representación de individuos. Los barrales de este tipo no presentan vertedera y los cuellos tienen tendencia a ser más alargados.

De manera análoga, para la forma puchero - ansat-, la correlación de las variables diámetro máximo y altura determinan con precisión una gradación de tamaños bien determinada (Fig. 5h). Por tanto, una comparación entre la distribución normal y la real que contempla todos los individuos en función del

\begin{tabular}{|c|c|c|c|c|c|c|c|c|c|c|c|c|c|c|}
\hline \multirow{2}{*}{$\begin{array}{l}\text { Tipo / tamaño } \\
\text { Alcuza }\end{array}$} & \multicolumn{2}{|c|}{ diámBO } & \multicolumn{2}{|c|}{ diámBA } & \multicolumn{2}{|c|}{ diámMíN } & \multicolumn{2}{|c|}{ diámMAX } & \multicolumn{2}{|c|}{ gros orMíN } & \multicolumn{2}{|c|}{ grosorMÁX } & altura & \multirow{2}{*}{\begin{tabular}{|l|} 
perfil \\
57122
\end{tabular}} \\
\hline & 15,2 & $(-)$ & 180,0 & $(-)$ & 176,3 & $(-)$ & 351,2 & $(-)$ & 4,8 & $(-)$ & 10,1 & $(-)$ & 439,0 & \\
\hline Barreño & 763,6 & $(274,7)$ & 477,5 & $(328,1)$ & 554,7 & $(257,7)$ & 805,5 & $(281,8)$ & 20,7 & $(5,3)$ & 37,7 & $(7,8)$ & $825,8(315,0)$ & 06124 \\
\hline Barral (B1) & 52,6 & $(3,1)$ & 207,7 & $(25,3)$ & 41,5 & $(5,8)$ & 340,9 & $(7,9)$ & 5,7 & $(1,8)$ & 15,5 & $(4,0)$ & $408,2 \quad(15,8)$ & 44942 \\
\hline Mediano & 47,9 & $(8,4)$ & 151,1 & $(15,2)$ & 39,4 & $(4,3)$ & 258,6 & $(26,6)$ & 5,1 & $(0,4)$ & 10,8 & $(1,1)$ & $329,4 \quad(32,0)$ & 34832 \\
\hline Pequeño & 40,0 & $(2,3)$ & 111,9 & $(25,5)$ & 27,8 & $(1,8)$ & 199,7 & $(30,5)$ & 4,3 & $(0,1)$ & 10,2 & $(1,0)$ & $237,4 \quad(23,2)$ & 34832 \\
\hline Brasero & 100,5 & $(25,6)$ & 98,7 & $(28,5)$ & 98,8 & $(22,0)$ & 119,5 & $(90,5)$ & 4,5 & $(1,6)$ & 8,0 & $(1,7)$ & $114,2 \quad(26,9)$ & 46844 \\
\hline Cántaro de pescador & - & $(-)$ & 127,3 & $(20,3)$ & 65,7 & $(66,8)$ & 189,1 & $(4,7)$ & 4,8 & $(0,0)$ & 10,9 & $(1,4)$ & $170,2 \quad(28,1)$ & 86213 \\
\hline Cántaro de pozo & 112,8 & $(4,9)$ & 147,8 & $(2,4)$ & 125,7 & $(23,0)$ & 264,1 & $(18,1)$ & 4,8 & $(1,3)$ & 9,7 & $(4,8)$ & $307,7 \quad(75,4)$ & 36922 \\
\hline Jarra & 182,3 & $(3,6)$ & 166,6 & $(31,8)$ & 168,1 & $(3,5)$ & 350,2 & $(37,9)$ & 4,9 & $(0,1)$ & 12,8 & $(3,6)$ & $383,6 \quad(38,3)$ & 25922 \\
\hline Medida de aceite & 373,5 & $(-)$ & 185,8 & $(-)$ & 338,4 & $(-)$ & 367,6 & $(-)$ & 6,5 & $(-)$ & 14,1 & $(-)$ & 331,4 & 07913 \\
\hline Medida de vino & 134,3 & $(-)$ & 218,6 & $(-)$ & 111,3 & $(-)$ & 339,7 & $(-)$ & 6,1 & $(-)$ & 8,1 & $(-)$ & 369,5 & 34832 \\
\hline Olla alta & 183,5 & $(31,6)$ & 89,5 & $(15,5)$ & 169,6 & $(29,7)$ & 234,5 & $(41,0)$ & 4,9 & $(0,7)$ & 12,7 & $(1,3)$ & $218,6 \quad(38,7)$ & 13924 \\
\hline Grande & 208,6 & $(20,6)$ & 102,9 & $(4,3)$ & 192,6 & $(20,1)$ & 269,5 & $(14,6)$ & 4,6 & $(0,3)$ & 13,7 & $(1,1)$ & $250,4 \quad(16,8)$ & 13924 \\
\hline Mediana & 174,6 & $(18,1)$ & 75,1 & $(10,7)$ & 162,1 & $(17,4)$ & 223,2 & $(16,7)$ & 5,0 & $(0,9)$ & 12,3 & $(0,9)$ & $208,5 \quad(20,2)$ & 23924 \\
\hline Olla baja & 162,7 & $(30,7)$ & 80,9 & $(18,8)$ & 150,8 & $(26,7)$ & 230,6 & $(38,5)$ & 3,9 & $(0,6)$ & 11,1 & $(1,2)$ & $158,3 \quad(28,9)$ & 23835 \\
\hline Grande & 184,5 & $(8,6)$ & 96,3 & $(1,7)$ & 170,7 & $(4,7)$ & 261,1 & $(0,7)$ & 4,5 & $(0,7)$ & 12,3 & $(0,2)$ & 180,2 & 23925 \\
\hline Mediana & 166,6 & $(7,9)$ & 75,4 & $(22,1)$ & 153,0 & $(8,4)$ & 230,0 & $(22,5)$ & 3,6 & $(0,3)$ & 10,1 & $(0,3)$ & $157,5 \quad(24,5)$ & 23826 \\
\hline Pequeña & 111,2 & $(-)$ & 61,1 & $(-)$ & 106,4 & $(-)$ & 171,0 & $(-)$ & 3,6 & $(-)$ & 10,4 & $(-)$ & 116,1 & 34835 \\
\hline Olla castañera & 145,8 & $(11,4)$ & 80,1 & $(40,2)$ & 155,1 & $(13,7)$ & 232,7 & $(18,0)$ & 6,5 & $(1,2)$ & 14,6 & $(0,3)$ & $150,0 \quad(16,8)$ & 33837 \\
\hline Olla es férica & 153,3 & $(-)$ & 122,9 & $(-)$ & 153,2 & $(-)$ & 221,6 & $(-)$ & 4,2 & $(-)$ & 6,6 & $(-)$ & 205,6 & 14825 \\
\hline Puchero (A1) & 104,0 & $(19,9)$ & 60,2 & $(15,9)$ & 91,3 & $(16,8)$ & 121,9 & $(27,4)$ & 4,4 & $(0,6)$ & 7,7 & $(0,9)$ & $120,5 \quad(27,0)$ & 14913 \\
\hline Grande & 130,6 & $(-)$ & 87,1 & $(-)$ & 108,8 & $(-)$ & 163,5 & $(-)$ & 4,4 & $(-)$ & 7,9 & $(-)$ & 162,2 & 14922 \\
\hline Mediano & 119,2 & $(4,9)$ & 56,0 & $(13,7)$ & 104,3 & $(7,3)$ & 141,3 & $(8,2)$ & 4,5 & $(0,9)$ & 8,5 & $(0,8)$ & 139,3 & 14923 \\
\hline Pequeño & 101,4 & $(7,7)$ & 65,1 & $(11,8)$ & 89,9 & $(8,5)$ & 117,6 & $(9,2)$ & 4,4 & $(0,6)$ & 7,4 & $(1,0)$ & $116,0 \quad(12,8)$ & 13912 \\
\hline Muy реqueño & 73,3 & $(6,4)$ & 43,2 & $(6,4)$ & 65,8 & $(9,9)$ & 80,6 & $(2,3)$ & 4,0 & $(0,5)$ & 6,9 & $(0,3)$ & $(0,9)$ & 13912 \\
\hline Puchero (A2) & 128,5 & $(-)$ & 83,3 & $(-)$ & 114,6 & $(-)$ & 162,5 & $(-)$ & 5,2 & $(-)$ & 7,5 & $(-)$ & 183,2 & 14822 \\
\hline Tinaja & 211,0 & $(31,9)$ & 182,0 & $(3,6)$ & 180,4 & $(1,4)$ & 479,1 & $(9,0)$ & 6,4 & $(3,9)$ & 19,6 & $(5,9)$ & $481,0 \quad(25,5)$ & 36923 \\
\hline
\end{tabular}

FIG. 6. Tabla sumaria de los rasgos morfométricos definitorios de los principales tipos documentados en Quart. Para cada variable se indica el valor medio de las muestras (izquierda) y su desviación típica entre paréntesis (derecha). Se omite la desviación tipica cuando solo hay un ejemplar representado. Para los tipos en los que se han podido identificar tamaños estandarizados, se proporcionan los datos totales del tipo y desglosados por rango de dimensiones. 
diámetro máximo presenta una distribución bimodal muy pronunciada, con una discontinuidad marcada por la ausencia de individuos en el recuento entre $\mu-\sigma$ y $\mu-0,5 \sigma$, para una curva de distribución normal que presenta una variación menor que en el tipo anterior (Fig. 5i). Al comprobar nuevamente la correlación, pero solo para los tamaños pequeño y muy pequeño, la correlación negativa se corrige y la distribución pasa a ser unimodal y ajustada al valor medio, con una distinción entre ambos tamańos que se advierte en la discontinuidad del histograma (Fig. 5j). Vemos, pues, cómo los tipos presentan unos tamaños estándar que se mantienen estables en buena medida.

Estos ejemplos de validación de tipos presentan resultados positivos para todos los casos. Este hecho no es especialmente trascendente en el caso de piezas enteras, en el que la clasificación tipológica puede establecerse con facilidad a partir de la mera observación (Fig. 3). Sin embargo, un análisis morfométrico de este tipo realizado sobre una colección de piezas para las cuales conocemos la clasificación a priori permite testar la validez de la aproximación para aplicarla a un conjunto fragmentado, que son los más habituales en contextos arqueológicos, además de definir con precisión las características de cada tipo conocido (Fig. 6).

\subsection{Resultados de la clasificación morfométrica sobre un contexto fragmentado: la cerámica gris de Santa Margarida}

Para la realización de este análisis se ha tomado en cuenta todo el material procedente de cuatro silos con una secuencia relativa clara, cronológicamente distantes, y separados por algunas fases de arrasamiento de las estructuras de hábitat procedentes del yacimiento de Santa Margarida, en Martorell, Barcelona. La hipótesis de partida al abordar un intento de definición tipológica de estas características es que, a pesar de la homogeneidad de los materiales y de la dificultad que entraña identificar diferencias formales -más allá de una somera distinción visual entre ollas y lebrillos-, las variaciones existentes tienen una base cronológica, por lo que definir con claridad los tipos, más allá de las posibilidades que ofrece el examen visual, adscribirlos a una secuencia cronológica relativa y ponerlos en relación con los resultados del análisis de pastas y su caracterización petrográfica ${ }^{9}$ podía proporcionar un conocimiento más preciso de los patrones de cambio en los procesos de producción y distribución regional de la cerámica utilitaria de la zona (Travé et al., 2018).

La explotación estadística de las variables morfométricas consideradas permite conocer la estructura interna de los datos y definir con relativa facilidad una serie de grupos que, comparados con la representación gráfica de los materiales, ofrecen una tipología significativa en relación con los contextos estratigráficos. Un primer análisis de componentes principales $-\mathrm{ACP}-$, incluyendo la totalidad de las muestras y considerando todas las variables (Fig. 7a), permite hacer un primer balance del grado de fragmentación del conjunto y ofrece una distinción muy clara entre la forma olla $-\mathrm{O}-$ y lebrillo $-\mathrm{Ll}-$. Sin embargo, no posibilita aún la identificación de tipos, puesto que las piezas con una fragmentación menor $-\mathrm{y}$, por tanto, con unos valores de vectorización y altura muy distintos al resto- minimizan las diferencias entre piezas con un grado de fragmentación mayor y similar entre ellas. Son significativos los tres fragmentos - dos de olla y uno de cannata (c) - que componen el grupo GF-1, puesto que permiten recuperar más del $50 \%$ de la pieza completa, y los fragmentos de los grupos GF-2 y GF-3, que permiten recuperar un perfil significativo para cada individuo, superior al 30\% y $25 \%$ respectivamente de la altura total estimada, o bien recomponer piezas a partir de diversos fragmentos con forma atribuibles a un mismo individuo o, cuando menos, a un mismo tipo. El resto de formas globulares pertenecen a un cuarto grupo GF- 4 donde solo es posible considerar pequeños fragmentos de borde o base, con una altura inferior al $25 \%$ de la altura total estimada.

9 Travé, E.: Anàlisi petrogràfica de la Ceràmica Grisa procedent del jaciment de Santa Margarida (Sitges Q. XII). Informe inédito depositado en 2018 en el Centre d'Estudis Martorellencs. 

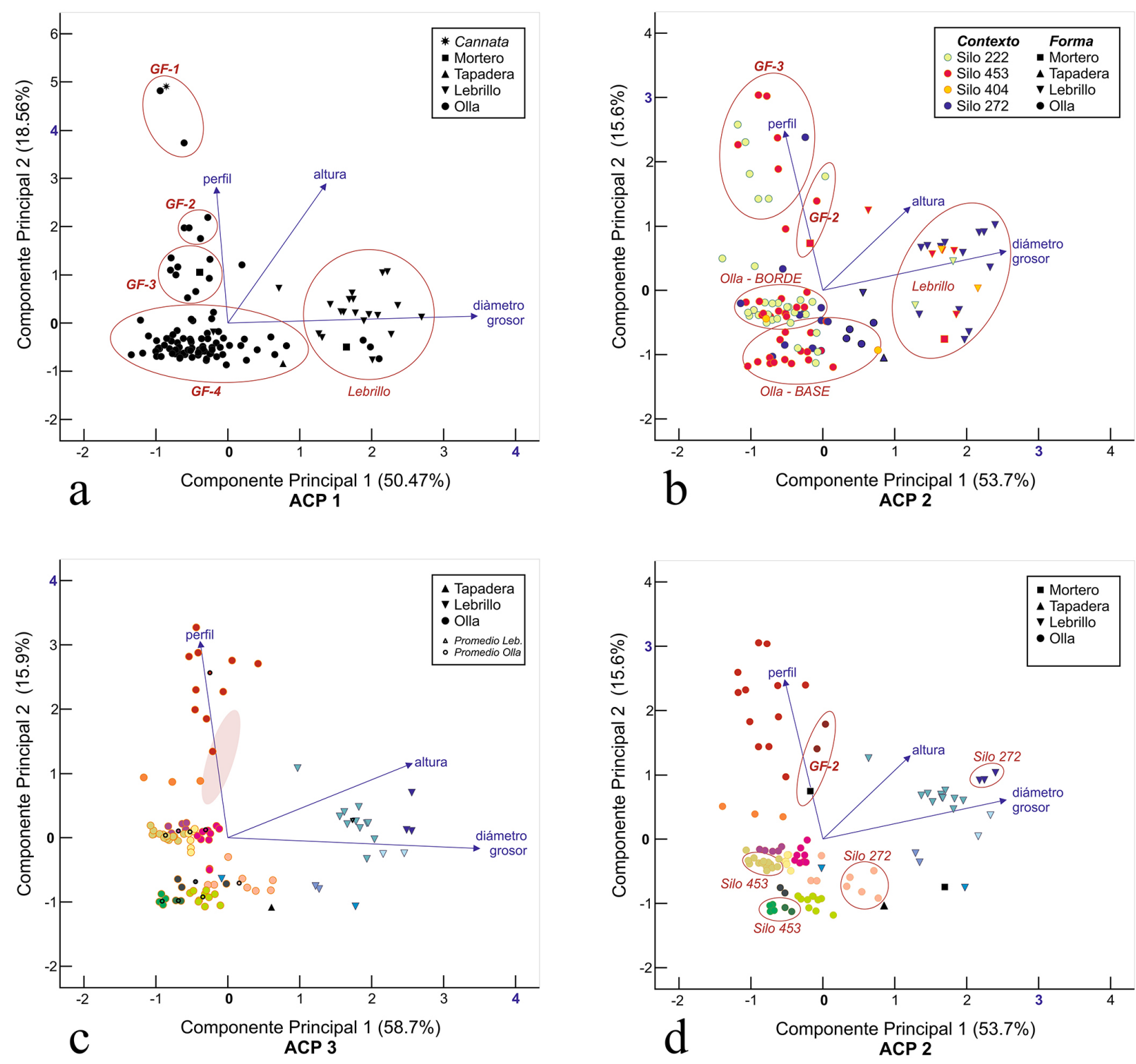

\begin{tabular}{|c|c|c|c|}
\hline \multicolumn{4}{|c|}{ GRUPOS MORFOMÉTRICOS } \\
\hline $\begin{array}{l}\text { Lebrillos: } \\
\nabla \text { Tipo A } \\
\nabla \text { Tipo B.1 } \\
\nabla \text { Tipo B.2 } \\
\nabla \text { Tipo C } \\
\nabla \text { Tipo D }\end{array}$ & $\begin{array}{l}\text { Ollas: } \\
\text { - Tipo A } \\
\text { Tipo B } \\
\text { Tipo C } \\
\text { - Tipo D.1 }\end{array}$ & $\begin{array}{l}\text { - Tipo D.2 } \\
\text { Tipo D.3a } \\
\text { Tipo D.3b } \\
\text { Tipo E }\end{array}$ & $\begin{array}{l}\text { - Tipo F.1 } \\
\text { - Tipo F.2a } \\
\text { - Tipo F.2b } \\
\text { - Tipo F.2c }\end{array}$ \\
\hline
\end{tabular}

Fig. 7. Gráficos de doble entrada que comparan el valor de los componentes 1 y 2 resultantes de los ACP practicados sobre los datos morfométricos de la cerámica procedente de los silos de Santa Margarida. Primer ACP, que permite identificar grupos de fragmentación, pero no tipos, con las muestras identificadas según la forma (a); segundo ACP calculado a partir de la omisión de GF-1, con las muestras identificadas según su forma y contexto, con los silos identificados a partir del $n .^{\circ}$ de UE correspondiente al recorte del mismo (b); tercer ACP calculado a partir de la omisión de GF-1 y 2 incluyendo el promedio de los tipos identificados según el segundo ACP (c); y de nuevo el segundo ACP, con las muestras identificadas en función de su forma y tipo (d). 
Por eso, tras una primera identificación de grupos de fragmentación -GF- entre el conjunto de ollas, se procede a la realización de dos nuevos ACP, uno de ellos excluyendo el GF-1 y otro excluyendo los grupos GF-1 y GF-2 (Fig. 7b-c). En ambos casos, la distinción entre ollas y lebrillos sigue siendo evidente, como es obvio, pero además resulta posible detectar algunas concentraciones de muestras susceptibles de ser analizadas en términos tipológicos en el seno de GF-4 y dichas concentraciones presentan una buena correlación entre ambos gráficos (Fig. 7c-d). La clasificación tipológica que presentamos ha sido realizada, por lo tanto, a partir de ambos análisis (Fig. 8). A pesar de lo acusada que es la fragmentación, el análisis morfométrico permite relacionar algunas formas de base y de borde susceptibles de ser consideradas como fragmentos representativos de un mismo tipo cerámico: véase la representación gráfica de los tipos O.D1 y O.F2B (Fig. 8), que representa unas ollitas medianas de las que conservamos bases y bordes. El cálculo de la vectorización del perfil en ambos casos, explotado junto con el resto de variables relativas al diámetro y altura, las agrupa de forma conjunta con independencia de que el fragmento conservado sea un borde o una base. Esta correlación entre bordes y bases no se produce siempre a partir de un ACP. Cuando las variables coincidentes susceptibles de ser relacionadas no son suficientes, la agrupación se ve afectada por la naturaleza del fragmento -borde o base-. En todo caso, es el analista quien, finalmente, puede establecer correlaciones en función de solo una o diversas variables. En el caso que nos ocupa, podríamos establecer una cierta correlación entre los grupos O.D2 y O.F1 en función del diámetro o bien entre O.D3в у O.F en sus distintas variantes también según el mismo parámetro. En cualquier caso, cuanto más elevada es la fragmentación menos fiable resulta la vinculación de tipos distintos.

$\mathrm{Al}$ margen de las formas globulares y su grado de fragmentación, que incluyen las formas olla y canna$t a$, debemos considerar un único grupo de fragmentación para el conjunto de lebrillos - Ll-. Los rasgos morfológicos de estos recipientes de forma abierta y paredes frecuentemente gruesas hacen que el grado

(C) Universidad de Salamanca de fragmentación no afecte especialmente la determinación de tipos, porque suele ser habitual la recuperación de un perfil que supera el 50\% de las dimensiones de la altura total estimada. La diferencia morfológica fundamental entre los tipos identificados a partir de la explotación estadística viene dada por el grado de concavidad de las paredes, con mayor tendencia esférica en el tipo Ll.A y prácticamente recta en el tipo Ll.B (Fig. 8). El tipo Ll.c se define como una forma intermedia entre las dos anteriores, con paredes ligeramente curvas y diámetros inferiores a los del tipo Ll.A. Es necesario destacar que esta tipología definida a partir de ACP ha permitido reconocer algunos fragmentos morfológicamente relacionados con una forma peculiar recuperada en el yacimiento, con una supuesta vertedera tubular adosada al borde que, por ahora, identificamos como un posible elemento auxiliar o receptáculo de algún tipo de tarea relacionada con la destilación o utilización de alambiques (Armengol y Lerma, 2012) y, por tanto, que no vierte nada, sino que canaliza la recepción de un líquido. Esta forma, identificada a partir de un único fragmento con este aplique cilíndrico junto al borde, cuenta con algún otro paralelo en el yacimiento, aunque nunca había sido posible, hasta ahora, identificarla al margen de su característico aplique. Sin embargo, la clasificación morfométrica incluyendo la variable de vectorización asocia esta forma al tipo Ll.D y la pone en relación con algunos otros fragmentos de morfología idéntica, algunos de los cuales permiten recuperar incluso perfiles completos, con un diámetro de base muy reducido. La convexidad de esta forma habría comprometido su estabilidad sobre una superficie plana, por lo que debemos considerar la posible utilización de algún tipo de apoyo o trípode. Los paralelos conocidos para estas piezas en Sant Cugat y Olèrdola (Roig, 2017) presentan una base plana y, que sepamos, no se les ha atribuido ninguna función concreta.

La distribución normal de los tipos así delimitados es poco pronunciada y las variables que habitualmente son determinantes del tamaño -diámetro máximo y altura- o no se conservan en todos los casos o se conservan de manera parcial y fragmentada. Si comparamos algunas de las variables con mayor o

Zephyrus, LXXXIV, julio-diciembre 2019, 161-182 




O.A


\section{LI.D}

FIG. 8. Tipología de ollas y lebrillos determinada para la cerámica de los silos de Santa Margarida a partir de los datos morfométricos, incluyendo la vectorización de perfiles. 
menor correlación para un tipo determinado (Fig. 9a-b) -como las variables diámetro de borde, diámetro mínimo y altura- para ollas del tipo в, que corresponden todas ellas al GF-3, puede observarse cómo las variables diámetro de borde y diámetro mínimo presentan una gran correlación, pero no es posible distinguir grupos con claridad en función de estas dos variables exclusivamente (Fig. 9a), aunque la distribución de medidas en el diámetro de borde sea bimodal (Fig. 9c) o la moda se aleje del promedio en el diámetro mínimo (Fig. 9d). Obviamente, la altura no es significativa en ningún caso, puesto que al tratarse de altura conservada y no de altura total depende del grado de fragmentación y, por tanto, su influencia en la determinación de grupos se relaciona con el perfil vinculado también a la fragmentación, como hemos comentado con anterioridad.
Del mismo modo, para un tipo -D3- determinado a partir de individuos muy fragmentados - GF4-, las mismas variables con un grado de correlación similar (Fig. 9e-f) permiten definir en algunos casos algunas variantes del tipo determinadas en función del tamaño. Al comparar las distribuciones normal y real de las variables diámetro mínimo y diámetro máximo vemos cómo una distribución normal muy similar se correlaciona bien con unas distribuciones reales unimodales en las que la moda se ajusta bien al promedio, sin superar el valor $\pm 0,5 \sigma$ (Fig. 9gh). La decisión de definir o no unas variantes del tipo en función del tamaño dependerá del grado de significación antropológica o histórica que pueda atribuírsele, que en nuestro caso no parece especialmente remarcable.
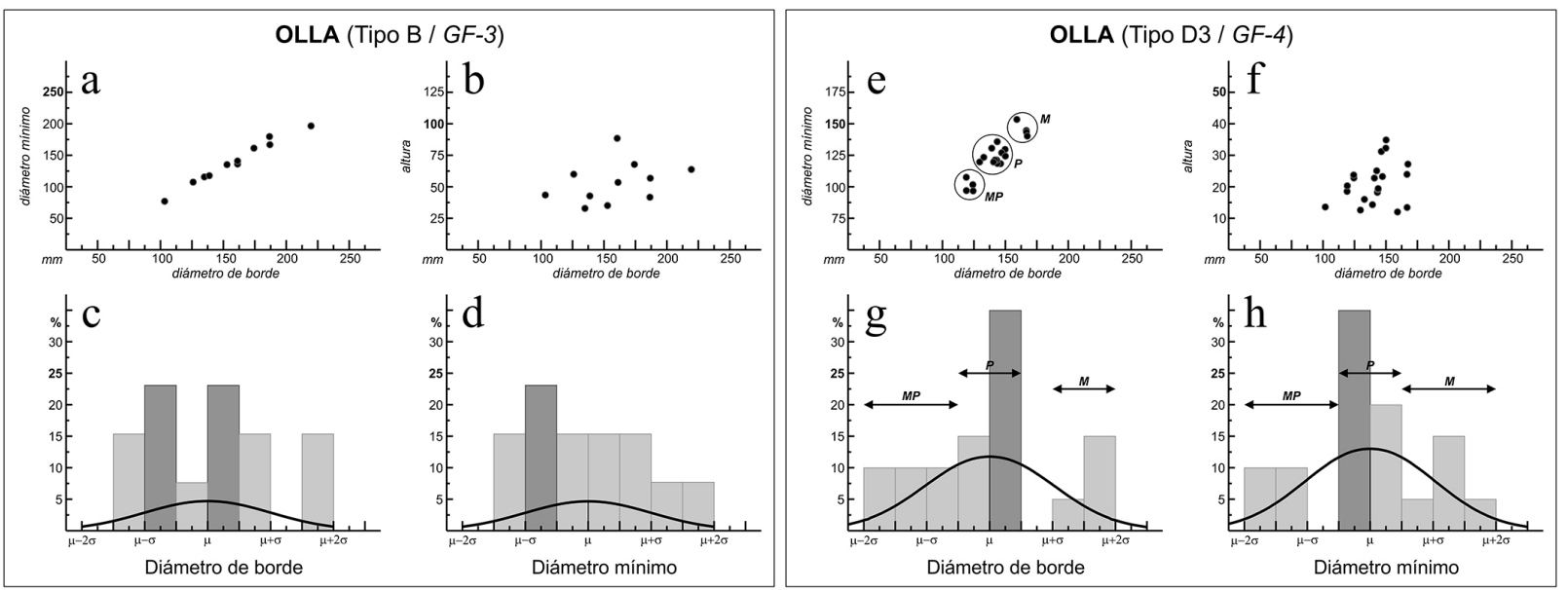

FIG. 9. Cuadro comparativo de las variables diámetro de borde, diámetro mínimo y altura en base al grado de correlación y su distribución normal e histogramas porcentuales absolutos para las ollas de los tipos B y D3 de Santa Margarida. Para cada uno de los tipos, gráficos bivariantes de la variable diámetro de borde respecto del diámetro mínimo (a, e) y altura $(b, f)$ respectivamente y distribuciones normales sobre los histogramas porcentuales absolutos para las variables diámetro de borde (c, g) y minimo $(d, h)$.

\section{Discusión. Algunas consideraciones sobre la definición de tipos}

La explotación estadística de datos morfométricos a partir de un análisis multivariante que incluya la forma del perfil, además de otros parámetros habituales como el diámetro, la altura o el grosor de las paredes, se revela como una herramienta útil para clasificar formalmente conjuntos numerosos de material. Las agrupaciones resultantes son satisfactorias con independencia del grado de fragmentación del conjunto, que habitualmente condiciona la identificación de formas cerámicas, especialmente cuando este es muy elevado. La razón fundamental para ello es el grado de correlación elevado que existe entre el valor calculado del perfil y otras variables habituales 


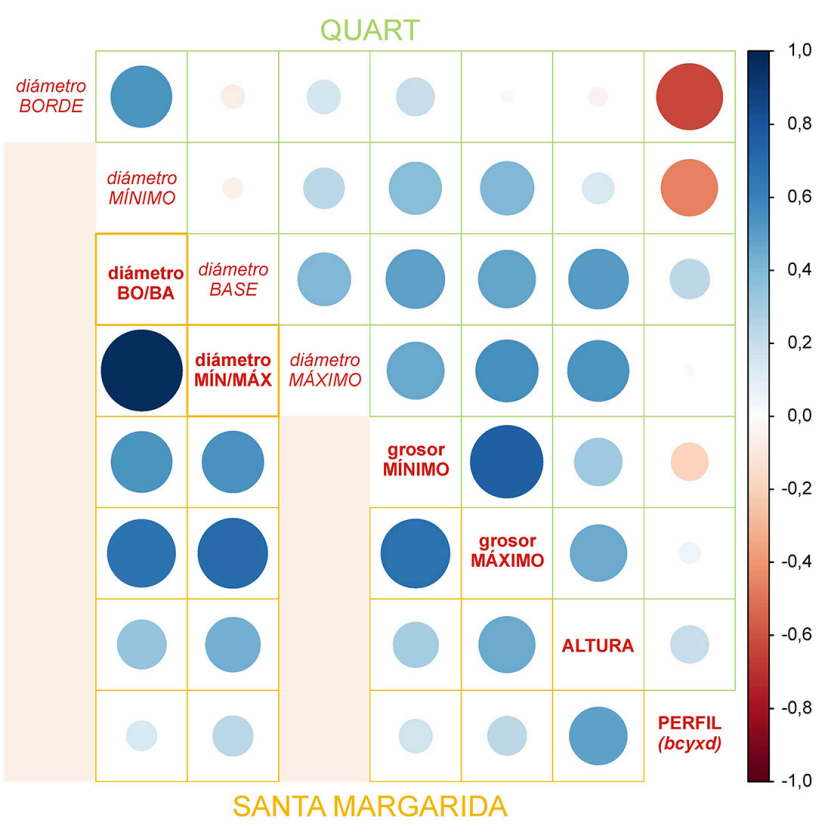

\begin{tabular}{|c|c|c|c|c|c|c|c|}
\hline \multicolumn{8}{|c|}{ QUART } \\
\hline $\begin{array}{l}\text { diámetro } \\
B O R D E\end{array}$ & 0,53 & $-0,07$ & 0,16 & 0,20 & 0,02 & $-0,05$ & $-0,63$ \\
\hline & $\begin{array}{l}\text { diámetro } \\
\text { MíNIMO }\end{array}$ & $-0,05$ & 0,24 & 0,39 & 0,41 & 0,14 & $-0,45$ \\
\hline & $\begin{array}{c}\text { diámetro } \\
\text { BO/BA }\end{array}$ & $\begin{array}{l}\text { diámetro } \\
B A S E\end{array}$ & 0,40 & 0,49 & 0,47 & 0,51 & 0,22 \\
\hline & 0,96 & $\begin{array}{l}\text { diámetro } \\
\text { MíN/MÁX }\end{array}$ & $\begin{array}{l}\text { diámetro } \\
\text { MÁXIMO }\end{array}$ & 0,46 & 0,55 & 0,54 & $-0,02$ \\
\hline & 0,54 & 0,55 & & $\begin{array}{l}\text { grosor } \\
\text { MíNIMO }\end{array}$ & 0,75 & 0,32 & $-0,20$ \\
\hline & 0,67 & 0,71 & & 0,69 & $\begin{array}{l}\text { grosor } \\
\text { MÄXIMO }\end{array}$ & 0,46 & 0,06 \\
\hline & 0,35 & 0,43 & & 0,29 & 0,46 & ALTURA & 0,20 \\
\hline & 0,13 & 0,23 & & 0,16 & 0,22 & 0,49 & $\begin{array}{l}\text { PERFIL } \\
\text { (bcyxd) }\end{array}$ \\
\hline
\end{tabular}

Fig. 10. Matrices de correlaciones comparadas - gráfica (izquierda) y numérica (derecha)-para los datos morfométricos de Quart y Santa Margarida en la parte superior e inferior de cada matriz respectivamente.

de medición, tanto en materiales enteros -Quartcomo fragmentados -Santa Margarida- (Fig. 10). En el caso de los materiales enteros, existe una correlación negativa muy elevada entre el perfil y los diámetros mínimo $(-0,45)$ o de borde $(-0,63)$. En el caso de los materiales fragmentados, la correlación entre la altura conservada y el perfil es mucho más elevada $-0,49$ frente a 0,20 en material entero-, por lo que ambas variables constituyen un buen indicador de los grupos de fragmentación, tal como hemos explorado para al caso de Santa Margarida.

Además, en contextos fragmentados, no se suele disponer de los cuatro diámetros calculados para el material entero, sino de los diámetros exclusivamente del borde o de la base y los respectivos diámetros mínimo o máximo, habitualmente bastante similares a los primeros, por lo que se reduce el número de variables explotables. El valor del diámetro mínimo o máximo en estos casos es real para el fragmento, pero aproximado en relación al tipo y no necesariamente coincidente con los del individuo completo en caso de que este se hubiera conservado. Esto explica en buena medida la correlación tan elevada $-0,96-$ a la par que poco significativa
(Fig. 9a-e) entre los diámetros de piezas que solo conocemos de manera parcial. A pesar de ello, lo significativo es que el grado de correlación mayor o menor entre las distintas variables es, hasta cierto punto, independiente del grado de fragmentación. Los grosores mínimos y máximos de las paredes presentan correlaciones elevadas en ambos casos, entre ellos y respecto de los diámetros, por lo que es especialmente interesante incluirlos como variables en los ACP, aunque no sean definitorios de tipos por sí mismos. De hecho, las variables con un grado de correlación medio, es decir, ni muy elevado $(=1)$ ni inexistente $(=0)$, que estimamos de manera genérica entre 0,4 y 0,6 , son especialmente significativas en la definición de tipos; valga como ejemplo la correlación entre el diámetro máximo y la altura en el caso de material entero - $0,54-$, que son las variables determinantes del tamaño de los vasos.

Considerar dimensiones habituales de los vasos como los diámetros, la altura y el grosor de las paredes, junto con una observación visual de la forma, parece suficiente para justificar la determinación de tipos al trabajar con materiales enteros o muy poco fragmentados, pero la inclusión de la variable del 
perfil aporta un valor ańadido considerable a dicha explotación. La curva que dibuja la generatriz del vaso - precisamente porque constituye uno de los elementos clave de la identificación formal- no parece descartable en un proceso de explotación estadística tal como proponemos en este estudio.

Otro aspecto que debemos considerar en base a los resultados obtenidos es la significación arqueológica o histórica de los tipos determinados de esta forma, ya sea en relación con aspectos sociales, funcionales, tecnológicos o cronológicos respecto a la producción y uso de la cerámica (Chang, 1967: 78). En el caso de Quart, la distinción morfológica se corresponde con las tipologías tradicionales conocidas, que tienen un significado funcional: son los alfareros quienes -en base a la tarea concreta para la que se debe utilizar el recipiente cerámico- producen una forma determinada de manera seriada que recibe un nombre específico e inalienable, marcadamente arraigado en la tradición, con un vocabulario propio de impacto regional reducido de muy difícil traducción (Associació de Terrissers Artesans de Quart, 2016).

La utilidad de la propuesta, sin embargo, debe demostrarse necesariamente en el caso de Santa Margarida, puesto que la muestra de Quart ha sido seleccionada de manera deliberada a fin de testar el método sobre una tipología conocida que, evidentemente, tenía significado por sí misma. La clave radica en dilucidar si, en el caso de Santa Margarida, en donde el conjunto recuperado en los silos es muy homogéneo y formado en su mayoría por lebrillos y ollas, los resultados son igualmente significantes. Cabe preguntarse si es necesario o útil distinguir entre los tipos O.D1, O.D3, O.E, O.F, etc., tratándose todos ellos de ollas más o menos esféricas con bases planas o ligeramente cóncavas (Fig. 8), por ejemplo. La comparación de los tipos obtenidos con el contexto arqueológico revela que, si bien no podemos atribuirles un significado funcional o tecnológico, sí que son significantes desde el punto de vista cronológico y, por tanto, es posible validarlos. Algunos de ellos son exclusivos en determinados contextos y parecen circunscritos a un periodo de utilización breve (Fig. $7 \mathrm{~b} / \mathrm{d})$.

(C) Universidad de Salamanca
Así, las ollas del tipo o.E, exclusivas del Silo 272, pueden considerarse más tardías que las del tipo oD.3, mucho más frecuentes en el Silo 222 y, por tanto, más antiguas. Del mismo modo, la mayor presencia de bordes que de bases en el Silo 222 y el número mayor de bases recuperadas en el Silo 453 son explicables en términos morfológicos: en el contexto del Silo 222 las ollas habrían sido mayoritariamente de cuerpo esférico, mientras que en el Silo 453 presentan fondos carenados que al romperse son más fácilmente reconocibles. Por ello el volumen de bordes estaría sobrerrepresentado en el primer caso, dado que los fondos esféricos sin carena no pueden distinguirse a priori de los fragmentos informes de pared (Travé, 2009: 311).

La incorporación de esta información de carácter tipológico al proceso de muestreo con finalidades arqueométricas como un elemento añadido al análisis macroscópico de las pastas ha permitido definir una muestra mucho más representativa del conjunto. Los resultados obtenidos, que serán objeto de publicaciones futuras, también son significantes en términos históricos, puesto que la precisión cronológica del muestreo a partir de la determinación de tipos ha permitido distinguir algunas producciones desgrasadas o micáceas más antiguas que desaparecen en un periodo de transformación del asentamiento (Travé et al., 2018) ${ }^{10}$.

En ambos casos, el análisis propuesto permite precisar con mayor exactitud los rasgos definitorios de cada tipo y resolver en cada caso las cuestiones inherentes a los problemas arqueológicos de fondo que suscitan cada conjunto de materiales. En el caso de Quart, una caracterización formal de este tipo permite estimar el alcance del grado de estandarización de los tamaños atestiguado por las fuentes escritas, mientras que en Santa Margarida permite determinar precisiones cronológicas en términos relativos a partir de su atribución estratigráfica. Los casos presentados ponen en relieve la utilidad de la vectorización del perfil en los términos propuestos para un estudio tipológico sobre una base analítica.

10 También Travé, op. cit. n. 9.

Zephyrus, LXXXIV, julio-diciembre 2019, 161-182 


\section{Conclusiones y perspectivas de futuro}

La definición y la construcción de tipologías arqueológicas en cerámicas comunes de época medieval y postmedieval en Cataluña presentan dificultades añadidas por el hecho de tratarse de materiales muy homogéneos, con variaciones muy escasas -aunque existentes- en periodos de tiempo muy dilatados y territorios muy amplios con circuitos de distribución regional. La búsqueda de estrategias que permitan incorporar un cierto grado de objetivación en los procesos de clasificación en la medida de lo posible nos ha llevado a definir y explorar el alcance de la explotación estadística de datos morfométricos. Incorporar a las variables habituales de diámetro, grosor y altura la variable de vectorización del perfil que hemos presentado en este trabajo y explotar estadísticamente el conjunto permite definir tipologías fiables a partir de la estructura interna de los datos, tal como hemos demostrado para los casos de Quart y Santa Margarida.

El análisis multivariante ofrece buenos resultados con independencia del grado de fragmentación a la hora de generar agrupaciones de carácter morfológico. En los conjuntos fragmentados permite además definir el grado de fragmentación y establecer diferencias morfológicas entre individuos de un mismo grupo en términos comparables. Gracias a ello, al cruzar la información obtenida con los grupos de pastas ha sido posible seleccionar una muestra mucho más representativa para una caracterización arqueométrica en curso de los materiales de Santa Margarida.

Consideramos que la propuesta es operativa en el marco de los procesos habituales de estudio de materiales arqueológicos puesto que siempre se contempla el dibujo de los materiales como una manera sencilla y esquemática de representar la forma y el tamaño de los vasos y de cuya exactitud dependen los análisis posteriores (Busto-Zapico, 2015: 207). Tanto si el dibujo se realiza de forma manual como si se automatiza por cualquier medio (Mauri et al., 2012: 59-62), la toma de medidas es un paso necesario e ineludible. El mero hecho de registrar estas variables durante el proceso de dibujo es lo que permite su explotación posterior.

(C) Universidad de Salamanca
El campo de estudio abierto alrededor de esta problemática es ingente y con nuestra contribución hemos pretendido simplemente llamar la atención sobre la necesidad de explorar las posibilidades que los métodos de análisis empleados en arqueometría, junto con las múltiples posibilidades que la tecnología pone a nuestro alcance, se contemplen en los procesos de construcción de tipologías. Aproximaciones de este tipo integradas en los protocolos habituales de análisis cuantitativo, especialmente en el campo de la cerámica gris y las producciones utilitarias sin decoración, deben contribuir a tener un conocimiento más específico de las distintas realidades alfareras en el territorio a partir de los siglos medievales.

\section{Bibliografía}

Adroher, A. M.; Carreras, C.; De Almeida, R.; FerNÁNDEZ, A.; Molina, J. y Viegas, C. (2016): “Registro para la cuantificación de cerámica arqueológica: estado de la cuestión y una nueva propuesta. Protocolo de Sevilla (prCs/14)", Zephyrus, LxxviII, pp. 87-110.

Artchinson, J. (1986): The statistical analysis of compositional data. Monographs on statistics and applied probability. London: Chapman and Hall.

Aitchison, J. y Greenacre, M. (2002): "Biplots for compositional data", Journal of the Royal Statistical Society, Series C (Applied Statistics), 51 (4), pp. 375392.

Arcelin-Pradelle, Ch. y Laubenheimer, F. (1985): "La notion de série en céramique tournée". En Histoire des techniques et sources documentaires. Méthodes d'approche et expérimentation en région Méditerranéenne. Cahier n. ${ }^{0}$ 7. Aix-en-Provence: Institut de Recherches Méditerranéennes.

Armengol, P. y Lerma, J. V. (2012): “Un conjunto de instrumentos cerámicos para la destilación de época califal procedente de Valencia”. En Gelichi, S. (ed.): Atti IX Congresso Internazionale sulla Ceramica Medievale nel Mediterraneo. Venezia: Al'Insegna del Giglio, pp. 372-374.

Associació de Terrissers Artesans de Quart (2016): Paraules que treballen el fang. Recull de mots i locucions dels oficis de Terrisser i Rajoler a Quart. Quart: Museu de la Terrissa de Quart-Cossetània Edicions.

Zephyrus, LXXXIV, julio-diciembre 2019, 161-182 
BaXter, M. J. (1995): "Standardization and Transformation in Principal Component Analysis, with Applications to Archaeometry", Journal of the Royal Statistical Society. Series C (Applied Statistics), 44 (4), pp. 513-527.

BaXter, M. J. (2006): "A review of supervised and unsupervised pattern recognition in archaeometry", $A r$ chaeometry, 48 (4), pp. 671-694.

Baxter, M. J. y Freestone, I. C. (2006): "Log-ratio compositional data analysis in archaeometry", $A r$ chaeometry, 48 (3), pp. 511-531.

Busto-Zapico, M. (2015): "Sobre el uso de herramientas informáticas para la cuantificación de cerámica: el EVE 2.0", SÉMATA. Ciencias Sociais e Humanidades, 27, pp. 199-219.

BuxedA, J. (2008): "Revisiting the compositional data. Some fundamental questions and new prospects in Archaeometry and Archaeology". En Daunis I Estalella, J. y Martín Fernández, J. (eds.): Proceedings of CO-DAWORK'08. The $3^{\text {rd }}$ compositional Data Analysis Workshop. Girona: Univ. de Girona, pp. 1-18.

Chang, K. C. (1967): Rethinking Archaeology. New York: Random House.

Contreras, F. (1984): "Clasificación y tipología en arqueología. El camino hacia la cuantificación”, Cuadernos de Prehistoria y Arqueología de la Univ. de Granada, 9, pp. 327-385.

Dillon, W. R. y Goldstein, M. (1984): Multivariate Analysis. Methods and Applications. New York: Wiley \& Sons.

EsCribano, S. (2017): "Estrategias cuantitativas para el estudio de cerámica arqueológica. Una propuesta desde el caso de la cerámica histórica alavesa", Munibe, 68, pp. 289-300.

García Jiménez, E.; Gil Flores, J. y Rodríguez GóMEZ, G. (2000): Análisis factorial. Cuadernos de Estadística. Salamanca: Hespérides.

Gilboa, A.; Karasik, A.; Sharon, I. y Smilansky, U. (2004): "Towards computerized typology and classification of ceramics", Journal of Archaeological Science, 31, pp. 681-694.

GonZÁlez, A. (ed.) (1997): La ceràmica medieval catalana. El monument, document. Quaderns científics i tècnics, 9. Barcelona: Diput. de Barcelona.

Hamilakis, A. y Jones, A. M. (2017): "Archaeology and Assemblage”, Cambridge Archeological Journal, 27 (1), pp. 77-84.

Karasik, A. y Smilansky, U. (2011): "Computerized morphological classification of ceramics", Journal of Archaeological Science, 38, pp. 2644-2657.
Llanos, A. y Vegas, J. I. (1974): Ensayo de un método para el estudio y clasificación tipológica de la cerámica. Vitoria: Diput. Foral de Álava.

Mateo, D. y Molina, J. (2016): “Archaeological Quantification of Pottery: The Rims Count adjusted using the Modulus of Rupture (MR)", Archaeometry, 58, pp. 333-346.

Mauri, A.; Travé, E. y Del Fresno, P. (2012): “An Integrated Implementation of Written and Material Sources. Conceptual Challenge and Technological Resources". En Ollich, I. (ed.): Archaeology. New approaches in theory and techniques. Rijeka: Intech, pp. 41-64.

NAIL, Th. (2017): "What is an assemblage?", SubStance, 46 (1), pp. 21-37.

Opgenhaffen, L.; Revello, M. y Kisjes, I. (2018): “Pottery goes public. Performing Archaeological Research Amid the Audience", Open Archaeology, 4, pp. 62-80.

Orton, C. (1988): Matemáticas para arqueólogos. Madrid: Alianza

Orton, C.; Tyers, P. y Vince, A. (1993): Pottery in Arhcaeology. Cambridge: cup.

Poblome, J. y Bes, Ph. (2018): "This is how we do it. Methodology of pottery processing at Sagalassos", Rei Cretariae Romanae Favtorvm Acta, 45, pp. 731-740.

R Core teAm (2018): $R$ : A language and environment for statistical computing. Viena: R Foundation for Statistical Computing. Disponible en https://www.R-project.org/.

Rıu, M. (ed.) (1984): Ceràmica grisa i terrissa popular de la Catalunya Medieval. Acta Mediaevalia, annex 2. Barcelona: Univ. de Barcelona.

Roig, J. (2017): "La cerámica reducida de cocina entre el Bajo Imperio Romano y la Alta Edad Media en el Noreste Peninsular (Cataluña)". En Obra Negra y alfarería de cocina. Actas XIX Congreso de la Asociación de Ceramología. Quart: Ayto. de Quart, pp. 63-122.

Sempere, E. (1985): La terrissa catalana. Tipologia i terminologia. Barcelona: Nou Art Thor-Diput. de Girona.

Solaun, J. L. (2005): La cerámica medieval en el País Vasco (ss. VIII-XIII). Sistematización, evolución y distribución de la producción. Vitoria-Gasteiz.

Soler, N. (1985): "Les càrregues de terrissa de Quart", Butlletí Informatiu de Ceràmica, 25, pp. 36-41.

Soler, N. (2009): "Ceràmica trobada als Banys Àrabs. La terrissa negra", Annals de l'Institut d'Estudis Gironins, 50, pp. 529-553.

Taiyun, W. y Simкo, V. (2017): R package "Corrplot": Visualization of a correlation Matrix (Version 0.84). Disponible en https://github.com/taiyun/corrplot.

Zephyrus, LXXXIV, julio-diciembre 2019, 161-182 
Travé, E. (2018): La ceràmica comuna de cuina d'època medieval: Provinença, tecnologia i comerç al camp català. Barcelona: Societat Catalana d'Arqueologia.

Travé, E. y Vicens, J. (2018): “Terrissa negra i canvi social: Pervivències i transformacions en l'ús de ceràmica de cocció reductora a Osona i les comarques gironines a partir de la recerca arqueològica i documental”, Ausa, 181-182, pp. 829-850.
VanPool, T. L. y LeOnard, R. D. (2011): Quantitative Analysis in Archaeology. Malden, MA: Wiley-Blackwell.

Vicens, J. y Travé, E. (2018): "La terrissa popular de Josep Escortell i Cerqueda: La tipologia de Quart", Estudis del Baix Empordà, 37, pp. 97-129.

Wiскнам, H. (2014): "Tidy Data", Journal of Statistical Software, 59/10, pp. 1-10. 